\title{
ORTHOMODULAR GENERALIZATIONS OF HOMOGENEOUS BOOLEAN ALGEBRAS
}

\author{
C. H. RANDALL, M. F. JANOWITZ* and D. J. FOULIS
}

(Received 31st August 1971)

Communicated by P. D. Finch

\section{Introduction}

It is well known that the so-called reduced Borel algebra, that is, the Boolean algebra of all Borel subsets of the unit interval modulo the meager Borel sets of this interval, can be abstractly characterized as a complete, totally non-atomic Boolean algebra containing a countable join dense subset. (For an indication of the history of this result, see, for example, ([2], p. 483, footnote 12.) From this characterization, it easily follows that the reduced Borel algebra $B$ is "homogeneous" in the sense that every non-trivial interval in $B$ is isomorphic to $B$.

In the present paper, we shall construct a class of orthomodular lattices ([1], p. 53), including the reduced Borel algebra $B$, with the following analogous property: If $L$ is any orthomodular lattice in this class, then every interval in $L$ is isomorphic to $L^{k}$ for some cardinal number $k$ (depending on the chosen interval).

The constructions set forth in this paper and the class of orthomodular lattices obtained as a consequence are of importance in connection with the program outlined in [8] aimed at providing a new approach to the logic of empirical science; however, this connection will not be emphasized here.

\section{The Free Orthogonality Monoid}

An orthogonality space is defined to be a pair $(X, \perp)$ consisting of a nonempty set $X$ and a symmetric, anti-reflexive binary relation $\perp$ defined on $X$. A classical orthogonality space is one of the form $(X, \neq)$. Suppose that $J$ is a nonempty indexing set and that for each $j \in J,\left(X_{j}, \perp_{j}\right)$ is an orthogonality space. Suppose further that $\left\{X_{j} ; j \in J\right\}$ is a pairwise disjoint family of sets. (If this were not so, one could replace $X_{j}$ by $\{j\} \times X_{j}$ and proceed in the obvious manner.) 11580.

* The research efforts of the second author were supported in part by NSF Grant GP 
Put $X=\cup_{j \in J} X_{j}$ and for $x, y \in X$ define $x \perp y$ if and only if for some $j \in J,\{x, y\} \subseteq X_{j}$ and $x \perp_{j} y$. Evidently $(X, \perp)$ is an orthogonality space, called the disjoint sum of the given family of orthogonality spaces. A semi-classical orthogonality space is defined to be a disjoint sum of classical orthogonality spaces.

Let $(X, \perp)$ be an orthogonality space and let $A \subseteq X$. We define

$$
A^{\perp}=\{x \in X ; x \perp a \text { for all } a \in A\},
$$

$A^{\perp \perp}=\left(A^{\perp}\right)^{\perp}$, etc. Evidently $A \subseteq A^{\perp \perp}, A^{\perp}=A^{\perp \perp \perp}$, and if $B \subseteq A$ then $A^{\perp} \subseteq B^{\perp}$. If $A=A^{\perp \perp}$ we say that $A$ is a closed subset of $(X, \perp)$. We denote by $\mathscr{C}(X, \perp)$ the set of all closed subsets of $(X, \perp)$. If $\mathscr{C}(X, \perp)$ is partially ordered by set inclusion, then it is clear that $\mathscr{C}(X, \perp)$ is a complete ortholattice ([1], p. 52) with the map $A \rightarrow A^{\perp}$ as its orthocomplementation. When there is no danger of confusion, we shall often write $\mathscr{C}$ in place of $\mathscr{C}(X, \perp)$.

Let $(X, \#)$ be a given orthogonality space and let $\Gamma$ be the free monoid (semigroup with unit 1) over $X$. Thus an element $b \in \Gamma$ may be considered to be a "word" $b=x y \cdots z$ whose "letters" $x, y, \cdots, z$ come from $X$ (with the understanding that the monoid unit 1 is regarded as being an "empty word"). If $x_{1}, x_{2}, \cdots, x_{n} \in X$ then we call the natural number $n$ the length of the word $x_{1} x_{2} \cdots x_{n}$ in $\Gamma$ and we write $\left|x_{1} x_{2} \cdots x_{n}\right|=n$. Of course we define $|1|=0$. The words in $\boldsymbol{\Gamma}$ are multiplied by simple juxtaposition; hence for $a, b \in \boldsymbol{\Gamma}$, $|a b|=|a|+|b|$. Naturally the words of length one in $\Gamma$ are identified with the corresponding elements of $X$, so that $X \subseteq \Gamma$. The orthogonality relation \# on $X$ can be extended naturally to an orthogonality relation $\perp$ on $\Gamma$ "lexicographically" as follows: For $a, b \in \boldsymbol{\Gamma}$, define $a \perp b$ if and only if there exist $c, d, e \in \boldsymbol{\Gamma}$ and $x, y \in X$ with $a=c x d, b=c y e$ and $x \not y$. The resulting orthogonality space $(\Gamma, \perp)$ is called the free orthogonality monoid over the base space $(X, \#)$.

Let $(\Gamma, \perp)$ be the free orthogonality monoid over the base space $(X, \#)$. If $A, B \subseteq \Gamma$ we define $A B=\{a b ; a \in A, b \in B\}$. We do not trouble ourselves over the distinction between an element $b \in \boldsymbol{\Gamma}$ and the singleton set $\{b\}$, so that for example, $A b$ means $A\{b\}$. We agree to call a word $w$ isolated in case $w^{\perp}=\varnothing$. Of course it may turn out that 1 is the only isolated word in $\Gamma$. For convenience in notation the letters $u, v, w$ will be reserved for isolated words; letters in the first part of the alphabet will denote arbitrary words in $\Gamma$, and the letters $x, y, z$ (with or without subscripts) will always denote elements of $X$. When we write $a=a_{1} a_{2} \cdots a_{n}$, however, it will be understood that each $a_{i} \in X$.

If $a, b \in \boldsymbol{\Gamma}$ we shall make frequent use of the fact that $(a b)^{\perp}=a\left(b^{\perp}\right) \cup a^{\perp}$. It follows for example that for $u$ isolated, $(a u)^{\perp}=a^{\perp}$. In view of this we may assume that for $a^{\perp \perp} \in \mathscr{C}, a$ is reduced in the sense that $a=a_{1} a_{2} \cdots a_{n}$ with $a_{n}$ 
a non-isolated element of $X$. In particular, then 1 is the only reduced isolated word in $\Gamma$.

We close this section with one final remark about notation. The meet operation in $\mathscr{C}$ is set intersection and will be denoted as such; the join operation, however, will be denoted by the symbol " $\vee$ ". We leave to the reader the routine verification that for $A, B \in \mathscr{C}, A \vee B=(A \cup B)^{\perp \perp}$.

\section{Standard Free Orthogonality Monoids}

By a standard free orthogonality monoid, we mean a free orthogonality monoid $(\Gamma, \perp)$ over a semi-classical base space $(X, \#)$. Those orthomodular lattices that are isomorphic to the lattice of closed subsets of some standard free orthogonality monoid comprise the class of orthomodular lattices referred to in the introduction.

For the remainder of this section it will be assumed that $(\Gamma, \perp)$ is a standard free orthogonality monoid over the base space $(X, \#)$. If $(X, \#)$ is the disjoint sum of a family of one element classical orthogonality spaces, then every word in $\Gamma$ is isolated and $\mathscr{C}$ is a two element Boolean algebra. For this reason it will be assumed that at least one of the classical orthogonality spaces in question has more than one element. Our goal will be to prove that $\mathscr{C}$ forms a complete atomless orthomodular lattice having the property that every interval of the form $\left.\mathscr{C}\left[\varnothing, a^{\perp \perp}\right)\right]$ is ortho-isomorphic to $\mathscr{C}$. We begin with a few technical results that will be used throughout the paper. We mention the fact that we in no way rule out the possibility that $(X, \#)$ be a classical space.

LEMMA 1. If $a$ is a reduced word in $\Gamma$ then $a^{\perp \perp}=a \Gamma$. It follows that $b^{\perp \perp} \subseteq a^{\perp \perp} \Leftrightarrow b \in a \Gamma$.

Proof. If $a=1$ there is nothing to prove, so we may assume that $a=c x$ with $x$ a non-isolated element of $X$. Evidently $a \Gamma \subseteq a^{\perp \perp}$. Assume $b \in a^{\perp \perp}$. If $y \in X$ is chosen so that $y \# x$ we then have $c y \in a^{\perp} \subseteq b^{\perp}$ so $b \in c\left(y^{\perp}\right) \cup c^{\perp}$. Now $b \perp c$ would force $b \perp a$, contrary to $b \in a^{\perp \perp}$. Thus $b \in c\left(y^{\perp}\right)$ so we may write $b=c z d$ with $y \# z$ and $d \in \Gamma$. If $z \neq x$ we would have $z \# x$. (Note the strong use of the fact that the base space $(X, \#)$ is semiclassical!) which would again lead to $b \in a^{\perp}$. We conclude that $z=x$ so that $b=c x d=a d \in a \Gamma$.

Lemma 2. Let $a, b \in \boldsymbol{\Gamma}$. Then:

(1) $a^{\perp \perp} \cap b^{\perp \perp} \neq \varnothing \Rightarrow a^{\perp \perp} \subseteq b^{\perp \perp}$ or $b^{\perp \perp} \subseteq a^{\perp \perp}$.

(2) Let $a^{\perp \perp} \cap b^{\perp \perp}=\varnothing$ with a not orthogonal to $b$. There exists a word $c \in \Gamma$ such that $a^{\perp \perp} \vee b^{\perp \perp}=c^{\perp \perp}$ and $|c|<\min (|a|,|b|)$.

Proof. The proof of (1) will be omitted as it follows immediately from Lemma 1 . We therefore concentrate on (2). We may clearly write $a=c x d$, 
$b=c y e$ with $c, d, e$ (possibly isolated) words in $\Gamma$ and $x, y$ distinct elements of $X$. Since $a$ is not orthogonal to $b$ we cannot have $x \# y$. The proof would be complete if we could establish that $c^{\perp \perp}=a^{\perp \perp} \vee b^{\perp \perp}$, and this is clearly equivalent to the assertion that $c^{\perp}=a^{\perp} \cap b^{\perp}$. Evidently $c^{\perp} \subseteq a^{\perp} \cap b^{\perp}$. If on the other hand, $g \in a^{\perp} \cap b^{\perp}$ then

$$
\begin{aligned}
& g \in a^{\perp}=c^{\perp} \cup c(x d)^{\perp} . \\
& g \in b^{\perp}=c^{\perp} \cup c\left((y e)^{\perp} .\right.
\end{aligned}
$$

Let $h \in(x d)^{\perp}$. If the first letter of $h$ is $x$, then $h \notin(y e)^{\perp}$; otherwise, $h \in z \boldsymbol{\Gamma}$ with $z \# x$. Since $(X, \#)$ is semi-classical we cannot have $z \# y$ and once again $h \notin(y e)^{\perp}$. Thus $(x d)^{\perp} \cap(y e)^{\perp}=\varnothing$. It follows that $c(x d)^{\perp} \cap c(y e)^{\perp}=\varnothing$ and from this that $g \in c^{\perp}$.

In the next theorem we gather up a few important facts about the lattice $\mathscr{C}(\Gamma)$.

TheOREM 3. $\mathscr{C}$ is a complete atomless orthomodular lattice. If the base space $(X, \#)$ is classical then $\mathscr{C}$ is a homogeneous Boolean algebra; otherwise $\mathscr{C}$ is simple but has the property that it is ortho-isomorphic to every interval of the form $\mathscr{C}\left[\phi, a^{\perp \perp}\right](a \in \boldsymbol{\Gamma})$.

For convenience the proof of the above theorem will be broken up into a number of individual "facts".

Fact A. $\mathscr{C}$ is a complete orthomodular lattice.

Proof. Since $\mathscr{C}$ is a complete ortholattice, it suffices ([1], p. 54) to show that for $M, N \in \mathscr{C}, M \subset N \Rightarrow N \cap M^{\perp} \neq \varnothing$. We begin by observing that $M \subset N \Rightarrow N^{\perp} \subset M^{\perp}$. Hence there is a reduced word $a \in M^{\perp} \backslash N^{\perp}$. Now $a \notin N^{\perp}$ forces the existence of a word $b$ of smallest length in $N$ such that $a$ is not orthogonal to $b$. Such a word is clearly reduced. The proof now breaks into three cases.

Case 1. $b^{\perp \perp} \cap a^{\perp \perp} \neq \varnothing$. Then $b^{\perp \perp} \cap a^{\perp \perp} \in N \cap M^{\perp}$ and we are done.

Case 2. $b^{\perp \perp} \cap a^{\perp} \neq \varnothing$. Let $c \in b^{\perp \perp} \cap a^{\perp}$. Then $c=b d$ for some $d \in \Gamma$. Since $a \perp b$ fails we must have $a \in b\left(d^{\perp}\right) \subseteq b^{\perp \perp}$. This puts $a^{\perp \perp} \subseteq b^{\perp \perp}$ and we are back in Case 1.

Case 3. $b^{\perp \perp} \cap a^{\perp \perp}=\phi=b^{\perp \perp} \cap a^{\perp}$. Then $c \in M \Rightarrow c \in a^{\perp}$ so $c^{\perp \perp} \subseteq a^{\perp}$ and $b^{\perp \perp} \cap c^{\perp \perp}=\varnothing$. If $b$ were not orthogonal to $c$ we could apply Lemma 2 to show that $b^{\perp \perp} \vee c^{\perp \perp}=e^{\perp \perp}$ with $|e|<|b|$. Now, $b, c \in N$ clearly implies $e \in N$, and we cannot have $e \perp a$, so this contradicts our initial choice of $b$. We conclude that $b \in N \cap M^{\perp}$.

Fact $\mathrm{B}$. Let $a \in \boldsymbol{\Gamma}$ be reduced. Then the mapping $M \rightarrow a M$ is an orthoisomorphism of $\mathscr{C}$ onto $\mathscr{C}\left[\phi, a^{\perp \perp}\right]$. 
Proof. We begin by observing that $a b \perp a c \Leftrightarrow b \perp c$. It follows that for $M \subseteq \Gamma, a\left(M^{\perp}\right)=(a M)^{\perp} \cap a^{\perp \perp}$. Hence

$$
\begin{aligned}
a\left(M^{\perp \perp}\right) & =\left(a M^{\perp}\right)^{\perp} \cap a^{\perp \perp}=\left[(a M)^{\perp} \cap a^{\perp \perp}\right]^{\perp} \cap a^{\perp \perp} \\
& =\left[(a M)^{\perp \perp} \vee a^{\perp}\right] \cap a^{\perp \perp}=(a M)^{\perp \perp} .
\end{aligned}
$$

Thus $M \in \mathscr{C} \Rightarrow a M \in \mathscr{C}\left[\varnothing, a^{\perp \perp}\right]$. Evidently $M \subseteq N \Leftrightarrow a M \subseteq a N$ so we need only show that the mapping in question is in fact onto. If $M \subseteq a^{\perp \perp}$ is closed, then by Lemma $1, M \subseteq a \Gamma$. Hence if $N=\{b ; a b \in M\}$ we have $a N=M$ and so $a\left(N^{\perp \perp}\right)=M^{\perp \perp}=M$.

Fact C. $\mathscr{C}$ is atomless.

Proof. Any atom of $\mathscr{C}$ must clearly be of the form $a^{\perp \perp}$ with $a \in \Gamma$. Now apply Fact B.

Fact D. If the base space $(X, \#)$ is not classical then $\mathscr{C}$ is simple.

Proof. It suffices to show that for each non-isolated word $a \in \Gamma, a^{\perp \perp}$ and $a^{\perp}$ have a common complement in $\mathscr{C}$. This we shall now establish. If $X$ has no isolated letters we may write $a=x d$ with $d \in \Gamma$. Choose $y \in X$ so that $x \# y$ fails and let $b=y$. On the other hand, if $X$ has an isolated letter $u_{1}$, we may write $a=u x d$ with $x \in X$ non-isolated and take $b=u u_{1} x$. In either case we have found a word $b$ not orthogonal to $a$ such that $a^{\perp \perp} \cap b^{\perp \perp}=\varnothing$ and $a^{\perp \perp} \vee b^{\perp \perp}=\Gamma$. If $c \in b \Gamma$ then $c \perp a$ fails, so $a^{\perp} \cap b^{\perp \perp}=\varnothing$. Similarly, $a^{\perp \perp} \cap b^{\perp}=\varnothing$ so $a^{\perp} \vee b^{\perp \perp}=\Gamma$. This shows $b^{\perp \perp}$ to be the desired common complement for $a^{\perp \perp}$ and $a^{\perp}$.

Fact $E$. If the base space $(X, \#)$ is classical then $\mathscr{C}$ is a homogeneous Boolean algebra.

Proof. Let $(X, \#)$ be classical. It turns out that the lattice $\mathscr{C}$ is of a type that has been studied in some detail by Pierce in [7]. To see this, we present herewith a portion of Pierce's construction. Let $\Phi$ be the set of all functions $\phi$ from finite (possibly empty) subsets of the positive integers to $X$, together with a zero symbol 0 . Partially order $\Phi$ by the rule $0 \leqq \phi$ for all $\phi$ and $\phi \leqq \psi$ if the domain of $\psi$ (in symbols, $D(\psi)$ ) is contained in the domain of $\phi$ and $\left.\phi\right|_{D(\psi)}=\psi$. As in [7], p. 131, $\Phi$ is a disjunctive semilattice and can therefore be imbedded as a dense subsemilattice of a complete Boolean algebra ([6], Theorem 3.3 , p. 6). This Boolean algebra is determined up to isomorphism by $\Phi$ and (following Pierce's notation) will be denoted $B_{\alpha \alpha \gamma}$ where $\alpha=\aleph_{0}$ and $\gamma$ denotes the cardinality of the set $X$. Furthermore, by [7], Lemma 3.12, p. 136, $B_{\alpha \alpha \gamma}$ is homogeneous. Our goal will be to show that $B_{\alpha \alpha \gamma}$ is isomorphic to $\mathscr{C}$.

An easy induction argument shows that for $a, b \in \Gamma a^{\perp \perp} C b^{\perp \perp}$ in the sense of [1], p. 52. It follows from [1], Theorem 23, p. 53 that $\mathscr{C}$ is a Boolean algebra. 
Let us adjoin a zero symbol 0 to $\Gamma$ and partially order the resulting set $\boldsymbol{\Gamma}_{0}=\boldsymbol{\Gamma} \cup\{0\}$ by the rule $0 \leqq a$ for all $a \in \boldsymbol{\Gamma}$ and for $a, b \in \Gamma, a \leqq b \Leftrightarrow a^{\perp \perp} \subseteq b^{\perp \perp}$. Evidently $\boldsymbol{\Gamma}_{0}$ is a meet semi-lattice and the mapping $0 \rightarrow \varnothing, a \rightarrow a^{\perp \perp}$ imbeds $\Gamma_{0}$ as a dense subsemilattice of $\mathscr{C}$. In view of [6], Theorem 3.6, p. 7 we need only show that $\Gamma_{0}$ may be imbedded as a dense subsemilattice of $\Phi$. We define $F: \Gamma_{0} \rightarrow \Phi$ as follows: $F(0)=0 ; F(1)=$ the element of $\Phi$ having empty domain; if $a=a_{1} a_{2} \cdots a_{n} \in \boldsymbol{\Gamma}, F(a)$ is defined by the rule $F(a)(i)=a_{i}$ for $i=1,2, \cdots, n$. Evidently $F$ imbeds $\Gamma_{0}$ as a subsemilattice of $\Phi$ and we must show the imbedding to be dense. Accordingly, let $\phi \in \Phi$. There exists a positive integer $n$ such that $D(\phi) \subseteq\{1,2, \cdots, n\}$. Define $a_{i}=\phi(i)$ for $i \in D(\phi)$ and $a_{i}$ an arbitrary element of $X$ otherwise. Then $a=a_{1} a_{2} \cdots a_{n} \in \Gamma, D(F(a))=\{1,2, \cdots, n\} \supseteq D(\phi)$, and $\left.F(a)\right|_{D(\phi)}=\phi$. Hence $F(a) \leqq \phi$, thereby showing the imbedding to be dense.

COROLlaRY 4. The reduced Borel algebra is obtainable as $\mathscr{C}(\boldsymbol{\Gamma}, \perp)$ for a suitable standard free orthogonality monoid $(\Gamma, \perp)$.

Proor. Take as a base space a classical orthogonality space $(X, \neq)$ with $X$ a two element set. Then $\mathscr{C}(\boldsymbol{\Gamma}, \perp)$ will be a complete, atomless Boolean algebra containing a countable join-dense subset.

\section{Indexed Elements in $\mathscr{C}(\Gamma, \perp)$}

Let $L$ be an orthomodular lattice. An element $a$ of $L$ is called upper nearly central if $a \leqq b<1 \Rightarrow a$ central in $L[0, b]$ and lower nearly central if its orthocomplement $a^{\prime}$ is upper nearly central. If $b \in L$ is both upper and lower nearly central we say that $b$ is nearly central in $L$. The near center of $L$, in symbols $N C(L)$, is defined to be the set of all nearly central elements of $L$, and it has been shown [3], Theorem 9 that $N C(L)$ is a suborthomodular lattice of $L$. It is in fact clear from the proof of [3], Theorem 9, that $N C(L)$ is closed under the formation of any existing joins or meets in $L$.

A nonzero element $e$ of $L$ is said to be indexed in $L$ if there is a finite chain

$$
1=e_{0}>e_{1}>\cdots>e_{n}=e
$$

such that $e_{i}$ is nearly central in $L\left[0, e_{i-1}\right]$ for $i=1,2, \cdots, n$. Such a chain is called an indexing chain and it is said to have length $n$ if it contains $n+1$ distinct elements. By [3], Theorem 12, every indexed element $e$ can be connected to 1 by a unique indexing chain of minimal length. The length of this unique chain is called the index of $e$, and is denoted $K(e)$. By [3], Theorem 11, the indexing chain $1=e_{0}>e_{1}>\cdots>e_{n}=e$ is minimal if and only if $e_{i}$ is an atom of $N C\left[0, e_{i-1}\right]$ for $i=1,2, \cdots, n-1$. If this also holds for $i=n$, we say that $e$ is a minimally indexed element of $L$. The lattice $L$ is called (minimally) indexed if the (minimally) indexed elements are join-dense in $L$. 
If the base space $(X, \#)$ is a classical space, then $\mathscr{C}(\Gamma, \perp)$ is a Boolean algebra and the theory of indexed elements is trivial. Thus we assume for the remainder of this section that $(X, \#)$ is semiclassical but not classical. Our goal will be to prove that $\mathscr{C}(\boldsymbol{\Gamma}, \perp)$ is minimally indexed. We begin by characterizing the near center of $\mathscr{C}$. In connection with this it will be convenient to let $X_{0}$ denote the set of those words of the form $u x$ with $u$ an isolated word and $x$ a non-isolated element of $X$.

Lemma 5. $Q \subseteq X_{0} \Rightarrow Q^{\perp \perp}$ nearly central.

Proof. (1) We begin by showing $Q^{\perp \perp}$ to be upper nearly central. Let $B \in \mathscr{C}$ be such that $Q^{\perp \perp} \vee B \subset \Gamma$. We must show that $Q^{\perp \perp}$ commutes with $B$. This would follow if we could show that $u x \in Q, b \in B \rightarrow(u x)^{\perp \perp} C b^{\perp \perp}$. If $(u x)^{\perp \perp} \cap b^{\perp \perp}$ $\neq \varnothing$, them by Lemma 2 they are comparable elements of $\mathscr{C}$. If $(u x)^{\perp \perp} \cap b^{\perp \perp}=\varnothing$ and if they were not orthogonal, we would have by Lemma 2 that $(u x)^{\perp \perp} \vee b^{\perp \perp}=\Gamma$. This however, leads to the contradictory assertion that $Q^{\perp \perp} \vee B=\Gamma$. In either case, we have $(u x)^{\perp \perp} C b^{\perp \perp}$ as claimed.

(2) It is enough now to prove that $(u x)^{\perp \perp}$ is nearly central for all $u x \in X_{0}$. If $Y=\{y \in X ; x \not y\}$ then $(u x)^{\perp}=(u Y)^{\perp \perp}$. By $(1),(u Y)^{\perp \perp}$ is upper nearly central, so $(u x)^{\perp \perp}$ is nearly central.

LEMMA 6. The near center of $\mathscr{C}$ is atomic, and the atoms are those elements of the form $(u x)^{\perp \perp}$ with $u x \in X_{0}$.

Proof. Let $N$ be in the near center of $\mathscr{C}$ and $a \in N$. If $a=u x d$ with $x$ nonisolated, then $(u x)^{\perp \perp} \cap N \neq \varnothing \Rightarrow(u x)^{\perp \perp} C N$. Now $(u x)^{\perp \perp} \cap N^{\perp}$ is nearly central in $\mathscr{C}$, hence central in $\mathscr{C}\left[\phi,(u x)^{\perp \perp}\right]$. By Theorem $3, \mathscr{C}\left[\phi,(u x)^{\perp \perp}\right]$ is simple so we must have $(u x)^{\perp \perp} \cap N^{\perp}=\varnothing$ or $(u x)^{\perp \perp}$. Since $(u x)^{\perp \perp} \cap N \neq \varnothing$, we cannot have $(u x)^{\perp \perp} \subseteq N^{\perp}$. We deduce that $(u x)^{\perp \perp} \cap N^{\perp}=\varnothing$ and, since $(u x)^{\perp \perp}$ commutes with $N$, that $(u x)^{\perp \perp} \subseteq N$. If $\phi \subset M \subseteq(u x)^{\perp \perp}$ with $M$ nearly central, then $M \subseteq u x \Gamma$ and the above argument would show that $M=(u x)^{\perp \perp}$. This shows that $N C(\mathscr{C})$ is atomic and the atoms are precisely those elements of the form $(u x)^{\perp \perp}$ with $u x \in X_{0}$.

As an immediate consequence of Lemmas 5 and 6, we have

THEOREM 7. The near center of $\mathscr{C}$ is the set of all $Q^{\perp \perp}$ such that $Q \subseteq X_{0}$.

We are finally able to establish the main result of this section.

THEOREM 8. $\mathscr{C}(\Gamma, \perp)$ is minimally indexed.

ProOF. Note first that any reduced non-isolated word $a$ in $\boldsymbol{\Gamma}$ may be written in the form

$$
a=u_{1} x_{1} u_{2} x_{2} \cdots u_{n} x_{n}
$$


where $u_{1}, u_{2}, \cdots, u_{n}$ represent isolated words and $x_{1}, x_{2}, \cdots, x_{n}$ are non-isolated elements of $X$. Repeated applications of Fact B and Lemma 6 will now produce the fact that the chain

$$
\Gamma \supset\left(u_{1} x_{1}\right)^{\perp \perp} \supset\left(u_{1} x_{1} u_{2} x_{2}\right)^{\perp \perp} \supset \cdots \supset a^{\perp \perp}
$$

is a minimal indexing chain for the minimally indexed element $a^{\perp \perp}$. Since elements of this type are join-dense in $\mathscr{C}$, this completes the proof of the theorem.

\section{The Characterization of $\mathscr{C}(\Gamma, \perp)$}

We know from Theorems 3 and 8 that if $(X, \#)$ is a semiclassical orthogonality space that is not classical, then $\mathscr{C}(\Gamma, \perp)$ is a complete, minimally indexed orthomodular lattice such that for each $a \in \Gamma, \mathscr{C}\left[\phi, a^{\perp \perp}\right]$ is ortho-isomorphic to $\mathscr{C}$. Our goal in this section is to prove the converse of this result. The following lemma provides the key to what is involved.

LEMMA 9. Let $a, b$ denote minimally indexed elements of the orthomodular lattice $L$ and let

$$
\begin{aligned}
& 1=a_{0}>a_{1}>\cdots>a_{n}=a \\
& 1=b_{0}>b_{1}>\cdots>b_{m}=b
\end{aligned}
$$

denote minimal indexing chains. Then $a \perp b$ if and only if there exists a nonnegative integer $p \leqq \min (m, n)$ such that $a_{p} \perp b_{p}$ and $a_{i}=b_{i}$ for all $i<p$.

Proor. If such a $p$ were to exist, we would clearly have $a \perp b$. Let us therefore assume $a \perp b$. We cannot have $a_{i}=b_{i}$ for all $i \leqq \min (m, n)$ so let $p$ be the first integer at which they differ. Then $a_{p}, b_{p}$ are distinct atoms of the near center of $L\left[0, a_{p-1}\right]$. From the proof of [3], Theorem $6,0<a \leqq a_{p}$ with $a \perp b$ implies $b C a_{p}$, we so may apply [3], Lemma 8 to deduce that $a_{p} C b_{p}$, whence $a_{p} \perp b_{p}$.

At this point we ask the reader to recall the definition of disjoint sum. This has been given in several places (among them [3]), but for convenience we repeat it here. If $\left\{L_{\alpha} ; \alpha \in A\right\}$ is a disjoint family of orthomodular lattices with each $L_{\alpha}$ having more than two elements and the indexing set $A$ having cardinality at least 2 , we identify all of the zero elements with the symbol 0 , all of the unit elements with the symbol 1 , and let $L$ denote the union of the resulting family of sets. If $L$ is equipped with the partial order it inherits from the $L_{\alpha}$ 's, it forms an orthomodular lattice called the disjoint sum of the family $\left\{L_{\alpha} ; \alpha \in A\right\}$. In symbols we write $L=\boldsymbol{D S}\left(L_{\alpha} ; \alpha \in A\right)$. As an aid to understanding the terminology, we mention the easily established fact that if $(X, \perp)$ is the disjoint sum of the family of orthogonality spaces $\left(X_{\alpha}, \perp_{\alpha}\right)_{\alpha \in A}$ (with $A$ having cardinality at least 2) and if $L_{\alpha}=\mathscr{C}\left(X_{\alpha}, \perp_{\alpha}\right)$, then $\mathscr{C}(X, \perp)=\operatorname{DS}\left(L_{\alpha} ; \alpha \in A\right)$. 
THEOREM 10. Let L be a complete minimally indexed orthomodular lattice in which a minimally indexed implies $L[0, a]$ ortho-isomorphic to $L$. Then there is a semi-classical base space $(X, \#)$ that is not classical such that $L$ is ortho-isomorphic to $\mathscr{C}(\boldsymbol{\Gamma}, \perp)$.

Proof. If $L$ is a two element Boolean algebra, we may take $(X, \#)$ to be the disjoint sum of a family of one element classical orthogonality spaces, so we may as well assume that $L$ has more than two elements. It must therefore have a minimally indexed element $a<1$. It is immediate that $N C(L) \neq\{0,1\}$. If $0<z<1$ is nearly central, then $z \geqq a$ for some minimally indexed element $a$. If $1=a_{0}>a_{1}>\cdots>a_{n}=a$ is a minimal indexing chain, then $a_{1}$ is an atom of the near center of $L$, and since $z \wedge a_{1} \geqq a>0$ we must have $a_{1} \leqq z$. It follows that $N C(L)$ is atomic.

Suppose $L$ were reducible. By [3], Theorem 9 every nearly central element of $L$ would be central. Let $z_{1}$ be an atom of $C(L)$. Then $z_{1}$ is minimally indexed, so by hypothesis $L\left[0, z_{1}\right]$ is ortho-isomorphic to $L$. There must then exist an element $z_{2}<z_{1}$ such that $z_{2}$ is an atom of the center of $L\left[0, z_{2}\right]$. This implies $z_{2} \in C(L)$, a contradiction. We deduce that $L$ is irreducible, so by [3], Theorem 9 , we may write $L=\boldsymbol{D S}\left(L_{\alpha} ; \alpha \in A\right)$ with each $L_{\alpha}$ reducible. There is a corresponding decomposition of the near center of $L, N C(L)=\boldsymbol{D S}\left(N_{\alpha} ; \alpha \in A\right)$ with $N_{\alpha}=$ $C\left(L_{\alpha}\right)$ for each $\alpha \in A$. For $\alpha \in A$, let $X_{\alpha}$ denote the set of atoms of $N_{\alpha}$, and form the classical orthogonality space $\left(X_{\alpha}, \neq\right)$. Let $(X, \#)$ be the disjoint sum of this family of orthogonality spaces, and form the standard free orthogonality monoid $(\Gamma, \perp)$ over the base space $(X, \#)$. Our goal will be to prove that $L$ is ortho-isomorphic to $\mathscr{C}(\Gamma, \perp)$.

For each minimally indexed element $a$, let $f_{a}: L[0, a] \rightarrow L$ be a fixed orthoisomorphism. Let $I$ be the set of minimally indexed elements of $L$. Let $I$ be the set of minimally indexed elements of $L$. We define a mapping $f: I \rightarrow \Gamma$ as follows: $f(1)=1$. If $a<1$ is a minimally indexed element with $1=a_{0}>a_{1}>\cdots$ $>a_{n}=a$ its minimal indexing chain, let

$$
f(a)=a_{1} f_{a_{1}}\left(a_{2}\right) f_{a_{2}}\left(a_{3}\right) \cdots f_{a_{n-1}}\left(a_{n}\right) .
$$

There are one or two observations to be made at this point. First of all, $f_{a_{i-1}}$ is an ortho-isomorphism of $L\left[0, a_{i-1}\right]$ onto $L$. Since $a_{i}$ is an atom of the near center of $L\left[0, a_{i-1}\right]$, we have $f_{a_{i-1}}\left(a_{i}\right) \in X$ so $f(a) \in \boldsymbol{\Gamma}$ as claimed. In view of the uniqueness of the minimal indexing chain for $a$, the function $f$ is well defined.

We now define $g: \Gamma \rightarrow I$ by the rule: $g(1)=1 ; g\left(a_{1} a_{2} \cdots a_{n}\right)=$ the minimally indexed element $b$ whose minimal indexing chain is constructed as follows: Take $b_{1}=a_{1}, b_{2}=f_{b_{1}}{ }^{-1}\left(a_{2}\right), \cdots, b=b_{n}=f_{b_{n-1}}^{-1}\left(a_{n}\right)$. Then $1=b_{0}>b_{1}>$ $\cdots>b_{n}=b$ is a minimal indexing chain for the minimally indexed element $b$.

We observe that $f, g$ are mutually inverse mappings. But even more can be said. For let $a, b \in I$ with $a \perp b$. If 


$$
\begin{aligned}
& 1=a_{0}>a_{1}>\cdots>a_{n}=a \\
& 1=b_{0}>b_{1}>\cdots>b_{m}=b
\end{aligned}
$$

are the corresponding minimal indexing chains, then by Lemma 9 there exists a non-negative integer $p \leqq \min (m, n)$ such that $a_{p} \perp b_{p}$ and $a_{i}=b_{i}$ for $i<p$. Let $c=f\left(a_{p-1}\right), x=f_{a_{p-1}}\left(a_{p}\right)$ and $y=f_{a_{n}-1}\left(b_{p}\right)$. Note that $x, y \in X$ and that $a_{F} \perp b_{p}$ in $L$ implies that $x, y$ are distinct elements of some $X_{\alpha}$, so $x \# y$. It follows that $f(a) \perp f(b)$ in $\boldsymbol{\Gamma}$. Suppose conversely that $f(a) \perp f(b)$ in $\Gamma$. There must then exist $c, d, e \in \Gamma$ and $x, y \in X$ such that $x \not \# y$ and $f(a)=c x d, f(b)=c y e$. If $c=c_{1} c_{2} \cdots c_{k}\left(c_{i} \in X\right)$ it follows from the fact that $x \perp y$ in $L$ that $f_{c_{k}}^{-1}(x) \perp f_{c_{k}}{ }^{-1}(y)$, whence $a \perp b$. Thus $f$ is an orthogonality preserving bijection of $I$ onto $\Gamma$. Viewing $I$ as an orthogonality space with respect to the orthogonality it inherits from $L$, it is immediate that $\mathscr{C}(I)$ is ortho-isomorphic to $\mathscr{C}(\Gamma)$. By [5], Theorem 2.5, p. $601, L$ is ortho-isomorphic to $\mathscr{C}(I)$, and this completes the proof.

We mentioned in the introduction that we would be considering a class of orthomodular lattices with the property that for every lattice $L$ in the class, every interval in $L$ is ortho-isomorphic to $L^{k}$ for some cardinal number $k$. Our final goal will be to establish this fact for the lattices of the form $\mathscr{C}(\boldsymbol{\Gamma}, \perp)$ where $\boldsymbol{\Gamma}$ is a standard free orthogonality monoid. This will in fact follow from the following more general result.

LEMMA 11. Let $a$ be an element of the complete minimally indexed orthomodular lattice L. Call an element $b$ of L special if $b \leqq a$ and $b$ is minimally indexed with a minimal indexing chain $1=b_{0}>b_{1}>\cdots>b_{n}=b$ such that $b_{n-1} \leqq a$. Let $\left\{b_{2} ; \alpha \in A\right\}$ be the set of special elements of L. Then $L[0, a]=\prod_{\alpha \in A} L\left[0, b_{\alpha}\right]$.

ProOf. It clearly suffices to show that the special elements are pairwise disjoint central elements in $L[0, a]$ whose join is $a$. We note that $b_{\alpha} \leqq a$ and there is no minimally indexed element $c$ such that $b_{\alpha}<c \leqq a$. It follows from [3], Theorem 13 and [4], Lemma 1,29 that $\left\{b_{\alpha} ; \alpha \in A\right\}$ is an orthogonal family. If $d \leqq a$, we are to show that $d$ commutes with every $b_{\alpha}$. It is enough to establish this for $d$ minimally indexed. But this is clear, since then $d \leqq b_{\alpha}$ for some $\alpha \in A$. This shows each $b_{\alpha}$ to be central in $L[0, a]$. The proof is completed by noting that

$$
a \geqq V_{\alpha} b_{\alpha} \geqq \vee\{d ; d \text { minimally indexed, } d \leqq a\}=a
$$

THEOREM 12. Let $L$ be a complete orthomodular lattice. The following conditions are then equivalent:

(1) There exists a standard free orthogonality monoid $(\Gamma, \perp)$ over a nonclassical base space such that $L$ is ortho-isomorphic to $\mathscr{C}(\Gamma, \perp)$. 
(2) is minimally indexed and has the property that every interval of $L$ is ortho-isomorphic to some power of $L$.

(3) $L$ is minimally indexed and has the property that for every minimally indexed element a the interval $L[0, a]$ is ortho-isomorphic to $L$.

PROOF. (1) $\Rightarrow(3)$ This follows from Theorems 3 and 8 .

(3) $\Rightarrow$ (1) This is Theorem 10.

(3) $\Rightarrow$ (2) This is an immediate consequence of Lemma 11.

(2) $\Rightarrow$ (3) If $a \in L$ is minimally indexed, then $L[0, a]$ must be irreducible, hence ortho-isomorphic to $L$.

\section{References}

[1] G. Birkhoff, Lattice theory (Amer. Math. Soc. Colloq. Publ. XXV, 3rd edition, Providence, RI, 1967).

[2] A. Horn and A. Tarski, 'Measures in Boolean algebras', Trans. Amer. Math. Soc. 64 (1948) $467-497$.

[3] M. F. Janowitz, 'The near center of an orthomodular lattice', J. Austr. Math. Soc. 14 (1972), 20-29.

[4] M. F. Janowitz. 'Indexed orthomodular lattices', Math. Z. 119 (1971), 28-32.

[5] M. D. MacLaren, 'Atomic orthocomplemented lattices,' Pacific J. Math. 14 (1964), 597-612.

[6] R. S. Pierce, Translation lattices (Amer. Math. Soc. Memoir No. 32, 1959).

[7] P. S. Pierce, Some questions about complete Boolean algebras (Proceedings of Symposia in Pure Math, v. II, Lattice Theory, Amer. Math. Soc., Providence, RI, 1961), 129-140.

[8] C. H. Randall and D. J. Foulis, 'An approach to empirical logic'. Amer. Math. Monthly 77 (1970), 363-374.

University of Massachusetts

Amherst, MA 01002, USA 